\title{
Supplementary Comment on \\ "Neuropsychological Improvement in Patients With Cervical Spondylotic Myelopathy After Posterior Decompression Surgery"
}

This paper focuses on a specific problem, related, I think, to many chronic diseases; patients complain and present with significant reduction of neuropsychological performance, even though their disease (cervical myelopathy) does not affect the cerebral cortex. This fact has been demonstrated very well by Dr. Hoshimaru, applying a strict protocol: his patients (10 among a group of 79) presented with neuropsychological deterioration before surgery, that improved after decompression of the cervical spine. It is not possible to identify with certainty the reasons for this improvement, and the author advances some hypothesis. It could be just a fact related to the lack of attention to problems different from the specific disease that often accompanies chronic and severe illness. However, it could be worth remembering that the cortical organization changes in an impressive way after decompression for cervical myelopathy, as has been elegantly demonstrated recently by Holly et al. a few years ago. ${ }^{1)}$ These authors showed that the cortical areas devoted to body sensation become enormously enlarged during cervical compression, and may return to the normal small size after decompression. This in my opinion could affect also the neuropsychological performance. Therefore, I would encourage Dr. Hoshimaru to further study this interesting subject.

\section{Reference}

1) Holly LT, Dong Y, Albistegui-DuBois R, Marehbian J, Dobkin B: Cortical organization in patients with cervical spondylotic myelopathy. J Neurosurg Spine 6: 544-551, 2007

Alessandro Ducati, M.D. Ordinario di Neurochirurgia

Universita' di Torino Torino, Italy
This is an important paper, which deals with an underestimated harmful consequence of cervical spondylotic myelopathy: namely, decrease in cognitive functions. Ten of the 79 patients in the authors' series, i.e., $12.8 \%$, demonstrated clinically observable cognitive dysfunction, especially memory disturbances. In eight of the ten, SPECT measurements showed reduced regional CBF in the posterior cortical areas. After cervical decompression, neurophysiological test scores showed significant improvement $(\mathrm{p}<$ 0.05).This beneficial effect might well be due to reduction of pain and increase in physical activity, and also consequently to improvement of the reactional depressive state. However, physiological mechanisms could also play a role, as pointed out by the authors: 1) re-establishment of a better sympathetic regulation of CBS, and 2) restoration of a better CSF circulation in between the cranial and the rachidian compartments, both of them linked to recalibration of the cervical canal. Although we did not systematically study these phenomena in our series, we did noticed such beneficial, clinical and MR effects in a number of our patients. Further studies are of course needed to assess more objectively these physiological effects and in a larger number of patients. Also these studies might be extended to the field of Chiari I malformations.

Marc P. Sindou, M.D., Ph.D. Department of Neurosurgery Hôpital Neurologique P. Wertheimer Groupement Hospitalier Est Lyon, France

These are supplementary comments on an article published in Neurologia medico-chirurgica Vol. 50, No. 7, pp 554-559. 